\title{
DISCRETE SOLVABLE MATRIX GROUPS
}

\section{LOUIS AUSLANDER ${ }^{1}$}

Let $G L(n, R)$ denote the real general linear group with the usual topology. A subgroup of $G L(n, R)$ will be called an $n$-matrix group. We will say that a subgroup $G$ of $G L(n, R)$ is a discrete group if it is discrete in the induced topology. If for any group $G,[G, G]$ denotes the commutator subgroup of $G$, we will say that a group $S$ is solvable provided the sequence of groups $S=S_{1},\left[S_{1}, S_{1}\right]=S_{2}, \cdots$, $\left[S_{k-1}, S_{k-1}\right]=S_{k}$, terminates in the identity element for some $k$. If $S_{k}=e$ and $S_{k-1} \neq e$, where $e$ is the identity in $S$, then we will say that $S$ is $k$ step solvable and $k$ is called also the index of solvability. Then in [1] and [4] we have independent proofs of the following theorem:

TheOREM. Let $k(S)$ denote the index of solvability of $S$, where $S$ is a solvable n-matrix group. Then there exists an integer valued function of $n, f(n)$, such that $k(S)<f(n)$.

This note has as its purpose the proof of the following theorem:

THEOREM. Let $S$ be a discrete solvable $n$-matrix group. Then $S$ is finitely generated and, if \#(S) denotes the minimal cardinality of a set of generators for $S$, there exists an integer valued function $g(n)$ such that $\#(S)<g(n)$.

Proof. Theorem 1 in [1] tells us that there exists $S^{\prime} \subset S$ such that

(a) $S^{\prime}$ is of finite index, $I\left(S / S^{\prime}\right)$, in $S$.

(b) There exists a function $g_{1}(n)$ such that $I\left(S / S^{\prime}\right)<g_{1}(n)$.

(c) $S^{\prime}$ can be simultaneously triangulated over the complex field. Hence if we can now show that $S^{\prime}$ is finitely generated and \#( $\left.S^{\prime}\right)$ $<g_{2}(n)$ we would be done. But by Theorem 1 [3] $S^{\prime} \supset S^{\prime \prime}$ where $I\left(S^{\prime} / S^{\prime \prime}\right)<\infty$ and $S^{\prime \prime}$ is a fundamental group of a compact solvmanifold whose dimension is no more than $n(n+1) / 2$. By the structure theorem for the fundamental groups of solvmanifolds, we see that $S^{\prime \prime}$ is finitely generated and $\#\left(S^{\prime \prime}\right) \leqq n(n+1) / 2$. Hence all that we have to show is that $\#\left(S^{\prime} / S^{\prime \prime}\right)$ is a bounded function of $n$. But using the Theorem 1 of Wang and Theorem 3.1 of [2] we see that $S^{\prime} / S^{\prime \prime}$ is a discrete subgroup of torus of dimension $2 n$. Hence $\#\left(S^{\prime} / S^{\prime \prime}\right) \leqq 2 n$. This completes the proof of this theorem.

\section{REFERENCES}

1. A. Mal'cev, On certain classes of infinite solvable groups, Amer. Math. Soc. Translations, vol. 2, 1956, pp. 1-21.

Received by the editors March 30, 1960.

1 Partially supported by N.S.F. 
2. G. D. Mostow, Self-adjoint groups, Ann. of Math. vol. 62 (1955) pp. 44-55.

3. H. C. Wang, Discrete subgroups of solvable Lie groups I, Ann. of Math. vol. 64 (1956) pp. 1-19.

4. H. Zassenhaus, Beweis eines Satze über diskrete Gruppen, Abh. Math. Sem. Hansischen Univ. vol. 12 (1938) pp. 289-312.

INDIANA UNIVERSITY

\section{CONDITIONS IMPLYING CONTINUITY OF FUNCTIONS}

\section{EDWIN HALFAR}

In the study of functions on certain types of spaces, the question naturally arises as to what additional conditions may imply that the functions are continuous. Several papers, mainly $[2 ; 3 ; 4]$, have considered this problem. In this note, some further results of this type are developed.

To avoid repetition, a function $f$ will be at least on a Hausdorff space $X$ onto a Hausdorff space $Y$ with additional restrictions stated as needed. Also $f$ is compact preserving (connected) if when $K$ is a compact (connected) subset of $X, f(K)$ is a compact (connected) subset of $Y ; f$ has closed point inverses if for each $y \in Y, f^{-1}(y)$ is closed and $f$ is monotone if $f^{-1}(y)$ is connected. The rest of the terminology is standard.

In [1], it was shown that if $X$ is regular, $Y$ compact and if $f$ is closed with closed point inverses, $f$ is continuous. Combining this with Theorem 3.1 of [4], one has the result:

THEOREM 1. If $f$ is a closed monotone connected function on a regular space $X$ onto a compact space $Y$, then $f$ is continuous.

It is easy to see that without the assumption that $Y$ is compact, the conclusion need no longer hold.

THEOREM 2. If $X$ is locally compact, then if $f$ is compact preserving and point inverses are closed, $f$ is continuous.

Consider any point $x \in X$. Since $X$ is locally compact, $x$ has a neighborhood $U_{0}$ with a compact closure $\mathrm{Cl} U_{0}$. Because continuity is a local property, one need only consider $f$ restricted to $\mathrm{Cl} U_{0}$. On $\mathrm{Cl} U_{0}, f$ is closed and $f\left(\mathrm{Cl} U_{0}\right)$ is compact. Hence the conditions of Theorem 3 of [1] are satisfied and $f$ is continuous at $x$.

Received by the editors July 17, 1959. 